\title{
New Insights into the Role of Respiratory Inputs in Hippocampal Oscillations
}

\author{
(1) André L.V. Lockmann* and Hindiael Belchior* \\ Brain Institute, Federal University of Rio Grande do Norte, Natal, RN 59056-450, Brazil \\ Review of Viczko et al. and Yanovsky et al., 2014
}

The mammalian hippocampus generates oscillatory patterns as a result of synchronized activity of its neuronal populations. Over the last several years, it has been suggested that such rhythmic activity could have a mechanistic role for neural computations, constituting a temporal framework for neuronal communication (Buzsáki and Wang, 2012). Moreover, different hippocampal rhythms have specific behavioral correlates and have also been implicated in memory formation and retrieval (Buzsáki and Draguhn, 2004). Despite the growing interest in these oscillations, the mechanisms by which they are generated remain largely unknown. An important and yet unsolved question is to what extent hippocampal oscillations reflect pure endogenous network activity versus neuronal entrainment by afferent sensory inputs. Two studies recently published in The Journal of Neuroscience provide important new insights into the role of respiratory inputs in the generation of hippocampal oscillations (Viczko et al., 2014; Yanovsky et al., 2014).

Received May 7, 2014; revised June 2, 2014; accepted June 5, 2014.

The authors were supported by the Brazilian National Council of Technological and Scientific Development (CNPq) and the Brazilian Coordination for the Improvement of Higher Level Personnel (CAPES).

The authors declare no competing financial interest

${ }^{*}$ A.L.V.L. and H.B. contributed equally to this work.

Correspondence should be addressed to André L.V. Lockmann, Brain Institute, Federal University of Rio Grande do Norte, Av. Nascimento de Castro, 2155, Natal, RN 59056-450, Brazil. E-mail: andrelockmann@neuro.ufrn.br.

DOI:10.1523/JNEUROSCI.1870-14.2014

Copyright $\odot 2014$ the authors $\quad 0270-6474 / 14 / 349473-03 \$ 15.00 / 0$
During nonrapid eye movement (nREM) sleep and deep anesthesia, the rat hippocampus produces large-amplitude irregular activity and a more recently described slow oscillation (SO; lower than $1.5 \mathrm{~Hz}$ ) (Wolansky et al., 2006) that resembles the concurrent transitions between up and down states in neocortex (Steriade et al., 1993). Recording from ketamine-xylazine anesthetized rats, Fontanini et al. (2003) had previously shown that both the piriform cortex (PC) and the olfactory bulb (OB) exhibit slow oscillations in the local field potential (LFP) of similar frequency as the hippocampal SO; the slow oscillations in PC and OB, however, were clearly entrained by respiration rate. Based on this as well as on previous anatomical and functional data linking the hippocampus to olfactory processing (Vanderwolf, 1992), Viczko et al. (2014) addressed whether hippocampal SO could also result from the entrainment by inputs originated in the olfactory system.

Viczko et al. (2014) used multisite LFP recordings with simultaneous tracking of respiratory activity to analyze periods of SO in rats anesthetized with ketaminexylazine or urethane. In disagreement with the possibility raised above, the authors observed that SO recorded in CA1 was not significantly coherent with respiration; moreover, the peak frequency of SO and respiration differed. Consistent with Fontanini et al. (2003), however, Viczko et al. (2014) found field potentials in PC to be entrained by respiration cycle. Interestingly, there was significant coher- ence between LFPs recorded in CA1 and frontal cortex at the SO frequency. These results are in accord with Wolansky et al. (2006), who reported coherent slow oscillatory activity between the hippocampal fissure and neocortex along with no coupling between these regions and respiration (their supplemental Fig. 4). Of note, Viczko et al. (2014) found similar results during natural nREM sleep, although in this case there were no simultaneous recordings from the PC. Altogether, the results led Viczko et al. (2014) to conclude that SO in CA1, differently from the PC, is not entrained by olfactory inputs but is instead coupled to up and down transitions in forebrain structures during offline brain states.

A broader understanding about the role of olfactory inputs in hippocampal oscillations should take into account its anatomical and functional organization. The hippocampus has multilayered subregions that receive specific sets of inputs (Witter et al., 1989). Although Viczko et al. (2014) found no prominent coupling between CA1 and respiration, the latter may still impinge upon the activity of other hippocampal subregions. Indeed, the work of Vanderwolf (1992) suggests an anatomical specificity of sensorydriven oscillations in the hippocampus of freely behaving rats; namely, Vanderwolf (1992) reported that both odorants and electrical stimulation of the $\mathrm{OB}$ elicit fast oscillations $(15-30 \mathrm{~Hz})$ in the dentate gyrus (DG) but not in CA1. Similar fast oscillations have also been described in 
A

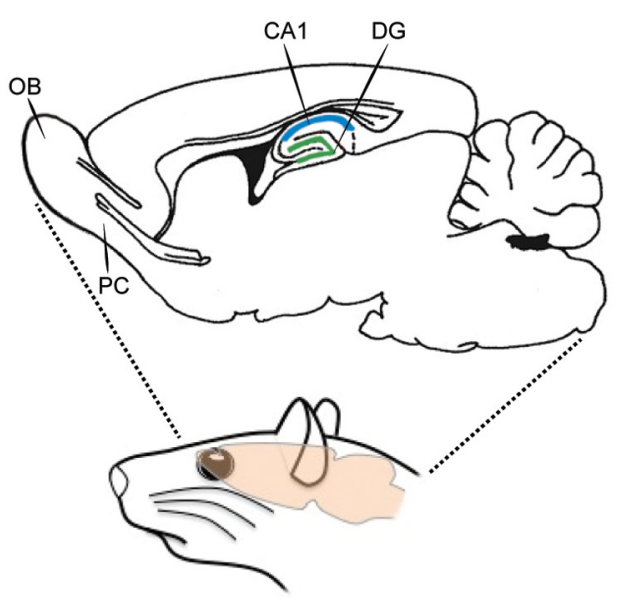

B

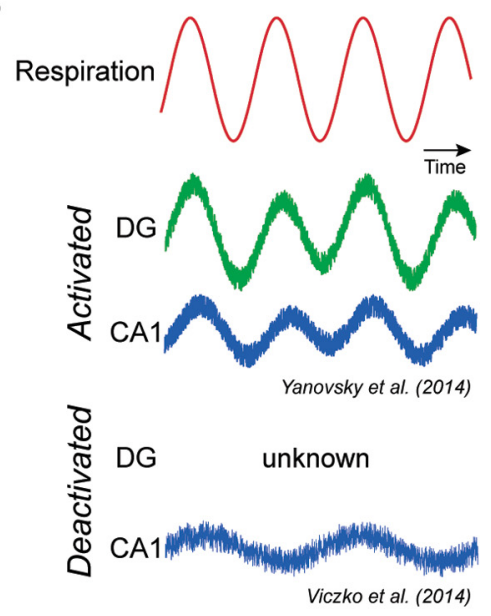

C
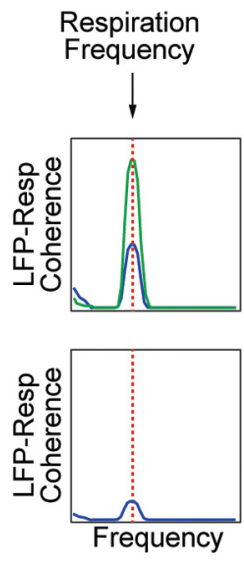

Figure 1. Schematic representation of current evidence of hippocampal entrainment by respiration. $A$, Sagittal section of the rat brain depicting brain regions in which LFP activity couples to respiration: $O B, P C, D G$ (green), and CA1 (blue). $B$, Representation of respiratory cycles (red) and concurrent LFPs in DG and CA1 during activated and deactivated states. DG oscillatory entrainment by respiration during deactivated states is unknown. C, Coherence spectra between DG and CA1 LFPs and respiration during activated and deactivated brain states under anesthesia.

OB and PC (Freeman, 1960; Bressler and Freeman, 1980). These olfactory structures project to the lateral entorhinal cortex (LEC), the major source of sensory information to the hippocampus via afferents to DG neurons (Steward and Scoville, 1976). Thus, in the same way as for fast oscillations, the slow respirationcoupled oscillations in OB and PC (Fontanini et al., 2003) would be more expected to drive network activity in DG than CA1. The pioneering work of Vanderwolf (1992), however, focused on respiration-induced beta and gamma oscillations, but did not investigate coupling at slower frequencies.

This issue was recently addressed by Yanovsky et al. (2014), who reported a novel type of hippocampal oscillation at the exact same frequency of respiration in urethane-anesthetized mice. Yanovsky et al. (2014) showed that this oscillation, dubbed hippocampal respiratory rhythm (HRR), is dependent on nasal respiration, since it disappears when mice breathe through tracheostomy. The influence of nasal respiration on hippocampal networks was not only evident in the LFP, but also in multiunit activity and membrane potential fluctuations of single neurons. In agreement with the anatomical specificity suggested by Vanderwolf (1992), HRR was much more prominent in DG hilus than CA1; coherence between HRR and OB LFPs as well as between HRR and respiration itself was also highest in DG. Notably, the authors showed that HRR modulates hippocampal gamma oscillations, and that the amplitude distribution of HRR across hippocampal layers per- fectly matches that of gamma, suggesting that the rhythms may share common inputs. Indeed, and in agreement with earlier reports (Wilson and Steward, 1978), Yanovsky et al. (2014) showed that electrical stimulation of afferents from either the OB or LEC yields prominent responses in DG, i.e., where both gamma and HRR have maximal amplitude.

Global brain states modulate the effectiveness of neuronal connections (Engel et al., 2001), and an important difference between the studies should be highlighted: Yanovsky et al. (2014) focused their analyses only on "activated" brain states, which are characterized by the emergence of theta oscillations and may occur spontaneously under light urethane anesthesia or-as used in their paper-be induced by tail pinching or brainstem stimulation; in contrast, Viczko et al.'s (2014) study mainly concerned periods of anesthesia in which the brain is considered "deactivated," characterized by slow-wave electrical activity similar to nREM sleep (Wolansky et al., 2006). Even though Viczko et al. (2014) did not find hippocampal-respiratory coupling during deactivated states, such coupling was present in the only analysis of activated periods under urethane (their Fig. 6A), just as reported inYanovsky et al. (2014) (their Fig. 4C). So, despite the paradoxical titles (i.e., Viczko et al. (2014) state that hippocampal slow oscillations are not coupled to respiration; Yanovsky et al. (2014) affirm that respiration leads to slow oscillations in the hippocampus), the results are actually complementary and even convergent when one takes into ac- count anatomical factors (CA1 vs DG) as well as the state-dependent dynamics of hippocampal networks (activated vs deactivated). One should also note that what is referred to as "slow oscillations" has different meanings in Viczko et al. (2014) (up- and down-like transitions, $<1.5$ ) and in Yanovsky et al. (2014) ( $\mathrm{HRR}, \sim 3.5 \mathrm{~Hz}$ ).

However, there are open questions that should be addressed to fully conciliate these results. For instance, Yanovsky et al. (2014) demonstrated the existence of HRR during activated states of anesthesia in mice, but did not investigate whether HRR is present or not in deactivated brain periods. Moreover, Viczko et al. (2014) did not investigate whether the lack of coupling between hippocampal LFPs and respiration during deactivated states in rats holds true for other hippocampal subregions in addition to CA1 (Fig. 1). It thus remains to be demonstrated whether the differences between the studies are solely due to methodological aspects or, although unlikely, due to physiological differences between the species studied (rats vs mice). On a technical note, mice and rats breathe at different frequencies, which should be taken into account when trying to isolate genuine respiratory rhythms from theta (in the case of mice) and up and down transitions (in the case of rats). Finally, and perhaps most importantly, it remains to be investigated whether respiration-induced rhythms occur in the hippocampus of freely behaving animals, and, if so, whether they play any functional role in linking olfaction and memory processes. 


\section{References}

Bressler SL, Freeman WJ (1980) Frequency analysis of olfactory system EEG in cat, rabbit, and rat. Electroencephalogr Clin Neurophysiol 50:19-24. CrossRef Medline

Buzsáki G, Draguhn A (2004) Neuronal oscillations in cortical networks. Science 304:19261929. CrossRef Medline

Buzsáki G, Wang XJ (2012) Mechanisms of gamma oscillations. Annu Rev Neurosci 35: 203-225. CrossRef Medline

Engel AK, Fries P, Singer W (2001) Dynamic predictions: oscillations and synchrony in top-down processing. Nat Rev Neurosci 2:704-716. CrossRef Medline

Fontanini A, Spano P, Bower JM (2003) Ketamine-xylazine-induced slow $(<1.5 \mathrm{~Hz})$ oscillations in the rat piriform (olfactory) cortex are functionally correlated with respiration. J Neurosci 23:7993-8001. Medline
Freeman WJ (1960) Correlation of electrical activity of prepyriform cortex and behavior in cat. J Neurophysiol 23:111-131. Medline

Steriade M, Nuñez A, Amzica F (1993) A novel slow $(<1 \mathrm{~Hz})$ oscillation of neocortical neurons in vivo: depolarizing and hyperpolarizing components. J Neurosci 13:3252-3265. Medline

Steward O, Scoville SA (1976) Cells of origin of entorhinal cortical afferents to the hippocampus and fascia dentata of the rat. J Comp Neurol 169:347-370. CrossRef Medline

Vanderwolf CH (1992) Hippocampal activity, olfaction, and sniffing: an olfactory input to the dentate gyrus. Brain Res 593:197-208. CrossRef Medline

Viczko J, Sharma AV, Pagliardini S, Wolansky T, Dickson CT (2014) Lack of respiratory coupling with neocortical and hippocampal slow oscillations. J Neurosci 34:3937-3946. CrossRef Medline
Wilson RC, Steward O (1978) Polysynaptic activation of the dentate gyrus of the hippocampal formation: an olfactory input via the lateral entorhinal cortex. Exp Brain Res 33: 523-534. Medline

Witter MP, Groenewegen HJ, Lopes da Silva FH, Lohman AH (1989) Functional organization of the extrinsic and intrinsic circuitry of the parahippocampal region. Prog Neurobiol 33:161-253. CrossRef Medline

Wolansky T, Clement EA, Peters SR, Palczak MA, Dickson CT (2006) Hippocampal slow oscillation: a novel EEG state and its coordination with ongoing neocortical activity. J Neurosci 26:6213-6229. CrossRef Medline

Yanovsky Y, Ciatipis M, Draguhn A, Tort AB, Brankačk J (2014) Slow oscillations in the mouse hippocampus entrained by nasal respiration. J Neurosci 34:5949-5964. CrossRef Medline 